\title{
"DE DIVISIONE REGNO": PODER MAGNATICIO EN LA SIERRA DE LA DEMANDA EN EL SIGLO XI
}

\author{
David Peterson \\ Universidad de Burgos
}

\section{Introducción}

Entre la rica y variada documentación medieval que se ha conservado en el Monasterio de San Millán de la Cogolla aparece un breve texto que destaca por su singularidad. Si la práctica totalidad del archivo emilianense se ocupa de las compraventas, donaciones, agregaciones y deslindes de los bienes inmuebles que acabarán componiendo el dominio del monasterio, aquí tenemos nada menos que un acuerdo delimitando la frontera entre el Reino de $\mathrm{Navarra}$ y el Condado de Castilla. A continuación reproducimos el texto, que se encuentra en el folio número 161 del Becerro Galicano del monasterio:

De divisione regno inter Pampilona et Castella, sicut ordinaverunt Sancio comite et Sancio regis Pampilonensem, sicut illis visum fuit una concordia et convenientia.

Id est de summa cuculla ad rivo Valle Venarie, ad Gramneto, ibi est molione sito et acollato Monnio, et a Biciercas et a Penna Nigra; deinde ad flumen Razon ubi nascit; deinde per medium monte de Calcanio, per summo lumbo et media Galaza, et ibi molione est sito, et usque ad flumen Tera, ibi est Garrahe antiqua civitate deserta, et ad flumen Duero.

Duenno Nunno Alvaro de Castella et sennor Furtun O ggoiz de Pampi-

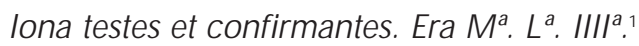

1. Texto tomado del Cartulario de San Millán de la Cogolla, ed. Antonio UBIETO ARTETA, Valencia, 1976, p. 161, documento número 166. En la elaboración de este trabajo, además del Cartulario de San Millán (en adelante 'Cogolla'), hemos utilizado las siguientes fuentes, con sus correspondientes abreviaturas:

- Albelda = Cartulario de Albelda, ed. Antonio U BIETO ARTETA, Valencia, 1960; 
En contraste con la singularidad y resonancia de la materia, el tamaño y formato del documento son muy modestos. Documentos de menor trascendencia, en cambio, se blindan con fórmulas notariales y se acompañan de largas listas de testigos. Creemos que esto puede ser un primer indicio de que este texto no sea todo lo que parezca a primera vista. Un segundo dato de importancia es el empleo en la redacción de dos tiempos verbales distintos: un pretérito indefinido en el primer párrafo (ordinaverunt ... fuit) y el presente (est) en el segundo. Más Ilamativo aun es el hecho de que el texto no se ocupa de la frontera en sí, sino de sólo una parte de ella. ${ }^{2}$

- Arlanza = Cartulario de San Pedro de Arlanza, ed. L. SERRAN O, Madrid, 1925;

- Burgos = Documentación de la Catedral de Burgos, ed. José M anuel GARRIDO, Burgos, 1983.

- Cardeña = Colección documental del Monasterio de San Pedro de Cardeña, ed. G. MARTÍNEZ DÍEZ, Burgos, 1998;

- Condes = Colección Diplomática de los Condes de Castilla, ed. M. ZABALZA, Valladolid, 1998.

- Irache = Colección Diplomática de Irache I (958 - 1222), ed. J. M . LACARRA, Zaragoza, 1965;

- Laturce = Documentación Medieval del Monasterio de San Prudencio de Monte Laturce (siglos X - XV), ed. J. GARCíA TURZA, Logroño, 1992;

- Leire = Documentación Medieval de Leire (siglos IX a XIII), ed. A. MARTíN DUQUE, Pamplona, 1983;

- Oña = Colección Diplomática de San Salvador de Oña (822-1284), ed. J. del ALAMO, Madrid, 1950;

- O ña2 = Documentación del Monasterio de San Salvador de O ña, ed. I. O CEJA, Burgos, 1983;

- Rioja = Colección Diplomática Medieval de la Rioja, ed. I. RODRÍGUEZ R. DE LAMA, Logroño, 1979;

- Sahagún = Colección Diplomática del Monasterio de Sahagún, Tomo II (1000 - 1073), ed. M. HERRERO DE LA FUENTE, León, 1988;

- Valpuesta = Cartulario de Valpuesta, ed. M. D. PÉREZ SO LER, Valencia, 1970;

- Valvanera = Documentación Medieval del Monasterio de Valvanera, siglos XI a XIII, ed. J. GARCÍA TURZA, Zaragoza, 1985;

2. Muchos investigadores han comentado este singular texto, pero pocos han reparado en las incongruencias que creemos detectar. Por ejemplo, Sánchez Candeira consideró a Nuño Álvarez el "hombre de confianza del conde Sancho García, por cuyo mandato había hecho la delimitación de fronteras entre Castilla y Navarra en el año 1016" (SÁNCHEZ CANDEIRA, A., Castilla y León en el siglo XI, Madrid 1999 (1950), p. 53), mientras U bieto Arteta resumiría el texto que nos ocupa como la "fijación de los límites entre el reino de Pamplona y el condado de Castilla", sin mencionar que el documento reproducido arriba no se ocupa de más que una parte de tales límites (UBIETO ARTETA, Cartulario de San Millán de la Cogolla, Valencia 1976, p. 161). El mismo autor, en su artículo "Las fronteras de Navarra" (Príncipe de Viana 14, 1953), a pesar del título del mismo dedica tan sólo una línea a este texto. Por su parte, García de Cortázar, quien se ha interesado más que nadie por la historia medieval altorriojana, tampoco repara en las incongruencias del documento, GARCÍA DE CORTÁZAR, J.A., El Dominio del Monasterio de San Millán de la Cogolla, Salamanca 1969, p. 141. Más recientemente, Zabalza Duque, en tantos otros sentidos tan acertadamente crítico, acepta que Nuño Álvarez "asiste como testigo y firma la fijación de límites entre Castilla y el Reino de Pamplona", y que el diplo- 
Para Martínez Díez la incongruencia entre la forma y el contenido de este texto se explica por el hecho de que no es más que una noticia, que resume un hipotético, y perdido, acuerdo más completo ${ }^{3}$. Estamos de acuerdo en que el texto reproducido no es el documento de factura real que traza la frontera entre los dos reinos, pero además sugerimos que el estudio de la cronología de las vidas y carreras de los dos confirmantes (Nuño Álvarez y Fortún O choiz ${ }^{4}$ ) demuestra que este texto es más que una mera noticia, siendo en realidad una ratificación parcial en el sentido espacial, probablemente posterior en términos cronológicos, y posiblemente particular en inspiración.

\section{El contexto espacial}

Primero, nos ocuparemos brevemente de la coordenada espacial, ya que nos parece evidente que el texto reproducido arriba se ocupa de tan sólo una parte de la frontera navarro-castellana de la primera mitad del siglo XI.

Cuando hacemos referencia en el título de este artículo a la Sierra de la Demanda, es en el sentido más amplio del topónimo, refiriéndonos, en realidad, a toda una serie de sierras del Sistema Ibérico que cubren la mitad meridional de la actual provincia de La Rioja y comarcas adyacentes de las provincias de Burgos, Soria e incluso Zaragoza. ${ }^{5}$

ma es una copia de "la divisoria de límites el año 1016, entre representantes del rey Sancho III el Mayor de Pamplona y el conde Sancho García", ZABALZA DU Q U E, Colección diplomática de los Condes de Castilla, pp. 526 y 578 respectivamente. Jimeno y Pescador reproducen el texto sin apenas comentario, cuando sí han sugerido cambios de datación para otros muchos diplomas, JIMENO ARANGUREN, R., y PESCADO R MEDRANO, A., Colección documental de Sancho Garcés III, el Mayor, rey de Pamplona (1004-1035), Pamplona 2003. Peña Bocos, quien ahonda más que otros en la figura de Fortún O choiz y en la extensión de su dominio, atribuye a ambos confirmantes "un poder de representación importante en los respectivos reinos", aceptando el contexto cronológico de 1016, aunque en otro momento sí le parece inquietar la longevidad del firmante riojano, PEÑ A BO COS, E., "Alfoces y tenencias: La Rioja", D el Cantábrico al Duero, (ed. García de Cortázar), Santander 1999, pp. 406-8, notas 84 y 79, respectivamente. El que más se ha acercado a nuestra lectura ha sido Isla Frez - "el documento tal y como se conserva [...] puede ser reelaboración posterior", ISLA FREZ, A., Realezas hispánicas del año mil, A Coruña 1999, p. 125.

3. MARTín EZ DÍEZ, G., "El Monasterio de San Millán y sus Monasterios Filiales. Documentación emilianense y diplomas apócrifos", Brocar 21 (1998), p. 42.

4. Así hemos homogeneizado a las numerosas ortografías alternativas que aparecen en la documentación consultada. También adoptamos la forma Don para representar las numerosas variaciones ortográficas (domno, duenno, dono etc.) utilizadas para este título. En el caso de Fortún 0 choiz el elenco de ortografías al ternativas es realmente impresionante: O choaz (Laturce2), Oggoiz (Cogolla166), Oggobiz (Cogolla179), Oscoa (Leire31), O ssoiz (Leire22), O ssuac (Leire20), O sxoa (Leire30), Oxoa (Rioja4), Oxoiç (Leire17), Oxoiz (Rioja3), Oxoriz (CogoIla256), Oxoys (O ña32), O ysgoac (O ña26), O ysgoaz (O ña19), Ozoiz (Cogolla146), Uxoaz (Albelda35), Uxoa (Albelda36), Uxoiz (Cogolla267), Uxoriz (Cogolla260), Uxuar (Albelda33), Uxuaz (Leire23), y U zoiz (0 ña35).

5. Desde las estribaciones septentrionales del Moncayo hasta el Pico de San Millán burgalés son más de cien kilómetros de orografía accidentada. Entre las denominaciones aplicables 
Los topónimos que contienen el documento parecen trazar una línea desde el Pico de San Lorenzo en La Sierra de la Demanda riojana, justo encima del monasterio de San Millán de "la Cogolla" (aquí cucullab), hacia la antigua y numantina Garray (Garrahe antiqua civitate deserta), en las inmediaciones de la actual ciudad de Soria. De los restantes topónimos, algunos son más difícilmente interpretados que otros, pero en general no hay gran dificultad a la hora de ver que se refiere a la zona entre estos dos puntos extremos: Valle Venarie -el actual Valvanera; Penna Nigra- acaso Montenegro de Cameros; flumen Razon -el río Razón (Soria); flumen Tera- el también soriano río Tera; y, por último, el flumen Duero, que no necesita ninguna explicación. Son poco más de 60 kilómetros en línea recta desde el Pico de San Lorenzo a Garray, y queda evidente que esto no es la totalidad de la frontera navarro-castellana del período.

Las comarcas que a lo largo de los siglos XI y XII serían disputadas por N avarra y Castilla (entre otras, los Montes de $\mathrm{O}$ ca, La Bureba, Las Merindades y la Cuenca de Miranda) no reciben mención en este breve texto. Sugerimos que la ausencia de un tratado equivalente para las (luego disputadas) comarcas noroccidentales se debe precisamente a la inconveniencia posterior de recordar antiguas y usurpadas (o modificadas, según el punto de vista) delimitaciones, pero la especulación sobre posibles causas de la no-supervivencia de un hipotético diploma no resulta muy fructífera. Lo que no se puede negar es que este tratado, tal y como nos ha llegado, delimita sólo una parte de la frontera entre Castilla y Navarra. ¿Por qué se eligió sólo a esta parte?

\section{Los confirmantes}

\section{a) Los dos Sanchos}

Se nombran a cuatro personas en el diploma: dos en un primer párrafo contextualizante y redactado en pretérito, que creemos referirse a un original (y ahora perdido) tratado que en probabilidad fijaría toda la frontera entre $\mathrm{N}$ avarra y Castilla; y otras dos que confirman y testifican el tratado parcial que nos ha sido conservado en el cartulario emilianense. Si en el primer párrafo se hace

a diferentes partes de este espacio se encuentran Tierra de Cameros, Sierra de la Demanda, Sierra de N eila, Picos de U rbión y Sierra Cebollera.

6. El orónimo Cuculla aparece todavía en la cartografía (Mapa Topográfico Nacional de España, 1:25.000, 240-IV "Valvanera") en el entorno del Cerro de San Lorenzo (N 42운 14', 0 $\left.2059^{\prime}\right)$, además de su recuerdo en relación con el célebre monasterio riojano. ZABALZA DU QUE (Colección diplomática de los condes de Castilla, p. 541) se confunde cuando sugiere que la frontera trazada arranca en el "alto de San Millán", 20 km. más al oeste.

7. Para análisis más detallado del trazado que suponen estos topónimos remitimos al lector al trabajo de MARTínEZ DÍEZ, G., Pueblos y alfoces burgaleses de la repoblación, Valladolid 1987, p. 201. Sin embargo, el interés principal de este artículo no es fijar precisamente la línea de frontera, sino demostrar que el texto conservado en el cartulario emilianense describe sólo la frontera parcial que separa a los dominios de los dos confirmantes. 
referencia a Sancio comite y a Sancio regis Pampilonensem, en el contexto de un tratado entre Castilla y Navarra con fecha de 1016, no dudaremos a la hora de identificar a éstos con el conde Sancho de Castilla (995-1017) y su yerno Sancho Garcés III, el Mayor, (1004-1035). Los problemas, sin embargo, empiezan cuando intentamos incorporar a los confirmantes Duenno Nunno Alvaro de Castella y sennor Furtun Oggoiz de Pampilona en esta cronología.

\section{b) Don Nuño Álvarez}

\section{i) La cronología de sus apariciones}

A parece Don Nuño Álvarez en este texto atípico con fecha de 1016, y además en un papel que sugiere singular importancia en el sector sur-oriental del condado castellano, pero, por lo demás, está ausente de la diplomática que hace referencia a ese espacio en ese período. En cambio, sí emerge tal combinación de nombre y patronímico con insistencia en la diplomática del período 1028-1065. Todo personaje histórico necesariamente tendrá una primera aparición en la documentación, pero que la primera mención esté tan separada cronológicamente de una, por lo demás, coherente serie de referencias que arrancan doce años después, y que además en esa primera referencia el personaje parece ostentar el máximo grado de importancia que alcanzará, es un patrón cuanto menos extraño.

\section{ii) El problema de la homonimia}

Existen serios problemas a la hora de reconstruir la biografía de cualquier individuo altomedieval, entre los más graves la penuria documental, pero en el caso de Nuño Álvarez el problema es otro, pues abundan las referencias a personas llamadas así en la diplomática castellana, con 86 casos entre 1028 y 1065. Además, en 18 ocasiones aparece más de un señor llamado Nuño Álvarez en un mismo documento, y en muchas ocasiones la repetición está explicitada al referir a uno de los dos como "alio" Nuño Álvarez. El problema radica en saber a cuál de los varios señores homónimos se está refiriendo en cada momento".

8. Don Nuño Álvarez es un personaje que ha atraído la atención de no pocos historiadores. Las razones detrás de este interés han sido esencialmente dos. Por un lado, un posible vínculo familiar con el Cid: MENÉNDEZ PIDAL, R., La España del Cid I, Madrid 1969, p.124; MARTín EZ DíEZ, G., El Cid Histórico, Barcelona 1999, pp. 42-3 y 47-8. Más relevante a la temática que nos ocupa, sin embargo, es el peso cuantitativo de la documentación que le menciona, lo cual le convierte en un paradigma para comprender la clase magnaticia castellana: ÁLVAREZ BO RG E, I., Poder y relaciones sociales en Castilla en la Edad Media, Valladolid 1996, pp. 83-88; PASTOR D ÍAZ DE GARAYO , E., Castilla en el tránsito de la antigüedad al feudalismo, Valladolid 1996, pp. 235-6 y 262; TO RRES SEVILLA, M., El Cid y otros Señores de la Guerra, León 2000, pp. 187-194; SÁN CHEZ DE M O RA, A., "La parentela de los Álvarez: un grupo gentilicio vinculado al territorio situado al sur del río Arlanzón (s. XI)", Silos. Un Milenio. Actas del Congreso Internacional sobre la abadía de Santo Domingo de Silos, Burgos 2003, pp. 441-455. 
Para ayudarnos en esta tarea disponemos de unas pocas referencias tópicas que contextualizan a los varios señores como de Aza, de Carazo, de Beniferi etc., y también el hecho de que aparecen juntos en la documentación varios hermanos Álvarez que mantienen cierta jerarquía entre sí en cuanto al orden en que firman los escatocolos. Pero nuestra arma más valiosa a la hora de seguir la pista del confirmante de Cogolla166 es el singular apelativo Duenno que ostenta. Conjugando estos varios elementos llegamos a la conclusión de que nuestro confirmante, D on N uño Álvarez, era de Carazo y tenía varios hermanos que también aparecen en la diplomática cortesana9. Entre 1033 y 1054 hay 16 referencias a un Don Nuño Álvarez, de un total de 52 referencias diplomáticas a los varios N uño Álvarez, pero a partir de 1054, la última aparición del título honorífico en este contexto, también desaparece de la diplomática la frase "alio Nuño Álvarez". En fin, sospechamos que Don Nuño Álvarez de Carazo murió ese año, muy posiblemente en la batalla de Atapuerca. Armados con el conocimiento de que Don Nuño era el de Carazo, y a la vez hermano de Diego y Fortún Álvarez, quienes le acompañan en tantas listas testificatorias, podemos establecer que, además de las 16 referencias explícitas que utilizan el apelativo domno $0^{10}$, un buen porcentaje de las restantes menciones hacen referencia a la misma persona, confirmante del diploma fronterizo que nos ocupa. ${ }^{11}$

No pretendemos haber desenmarañado todo el lío homonímico que envuelve a estos señores, pero sí creemos que se puede seguir la trayectoria de Don $N$ úñez con cierta confianza, y además se puede afirmar que, al margen de la problemática de la homonimia, esta combinación de nombre y patronímico se dan casi exclusivamente durante el segundo tercio del siglo XI, y no el primero.

En cuanto a la homonimia, Álvarez Borge analiza a los varios señores, todos ellos aparentemente de clase magnaticia, llamados N uño Álvarez, y la problemática de distinguir entre ellos.

9. Los diplomas O ña34 y 0 ña37 (1047 y 1054 respectivamente) son claves a la hora de reconstruir los hechos. Entre los hermanos de D on Nuño tenemos:

- Fortún, probablemente el segundo hijo, pues descontando a Don Nuño, Fortún precede a los otros hermanos en 21 ocasiones, Diego en doce, y Gonzalo tres veces. Desaparece de la diplomática a partir de 1054, quizás también víctima de la batalla de Atapuerca.

- Diego, luego tenente de O ca hasta c. 1085.

- Rodrigo, posible abuelo de Rodrigo Díaz de Vivar, muerto hacia 1082 (Burgos38).

- Gonzalo, hermano menor con presencia escatocolaria entre 1032 y 1075.

- y por lo menos una hermana, Mamadona (Arlanza54).

10. El principal criterio para la aparición de la denominación "domno" parece ser el grado de formalidad del diploma, ya que sus apariciones son siempre en documentos cortesanos, productos de diversas cancillerías reales, tanto castellanas como navarras e incluso en una ocasión aragonesa. En ningún caso se emplea el apelativo cuando el documento es de origen particular. Sobre el valor semántico del término vean PASTO R DÍAZ DE GARAYO, Castilla en el tránsito ..., pp. 232-6.

11. Sánchez de Mora ("La parentela de los Álvarez") también se inmersa en este grupo homonímico al considerar a los varios Álvarez del período, y a sus descendientes, como un clan magnaticio de suma importancia en las comarcas occidentales de Castilla. No obstante, no sigue la pista del apelativo 'domno', clave a la hora de distinguir entre los varios señores homónimos. 


\section{iii) El contexto geográfico}

Como ya hemos comentado, en dos ocasiones se relaciona a Nuño Álvarez explícitamente con Carazo. Aunque esta plaza dista aproximadamente 40 kilómetros de la línea fronteriza dibujada en Cogolla166, parece probable que el espacio intermedio fuera de escasa densidad demográfica, y sugerimos que la tenencia de Carazo bien podría ser uno de los más importantes centros de poder magnaticio de esta parte sur-oriental de la actual provincia de Burgos. Lo que no aclaran estas dos referencias es en qué sentido se relaciona a Don N uño con Carazo, ¿como delegado del poder central, o como magnate autóctono? En realidad las dos funciones no tendrían por qué ser incompatibles ${ }^{12}$, pero nos parece pertinente en este caso investigar la naturaleza de la relación entre estos magnates, sus zonas de influencia y el poder central.

La noticia (Arlanza54, 1052) de que, no sólo N uño, sino también sus hermanos Diego y Mumadona tenían posesiones en Carazo y en Lara, sugiere que su vínculo con la zona es en gran medida familiar y patrimonial, con el poderío político-militar como consecuencia y no causa. Esta distribución patrimonial, representada cartográficamente por Ernesto Pastor ${ }^{13}$, sugiere un núcleo alrededor del mismo Carazo, otros intereses en los llanos cerca de la desembocadura del Arlanza en el Arlanzón, pero también propiedades 25 kilómetros al este de Carazo, en Peñalba y en Vilviestre del Pinar ${ }^{14}$, así los bienes documentados reducen a la mitad la distancia entre la zona de influencia de N uño Álvarez y la frontera acordada con Fortún O choiz.

Su vinculación con la parte sur-oriental de Burgos se observa también en la radiografía de sus intervenciones diplomáticas. Aparece en 20 de los 26 documentos conservados en el archivo de San Pedro de Arlanza para el período 103754, y con algo menos de insistencia en el cartulario de Cardeña ${ }^{15}$. El marco geográfico de la mayoría de estas intervenciones depende, como cabría esperar, de los intereses del monasterio involucrado. Por lo tanto, aleja ligeramente a nuestro protagonista de la zona serrana donde sospechamos que tuviera la mayoría de sus propios intereses patrimoniales, en favor de espacios más Ilaneros entre la ciu-

12. "No hay, pues, que plantearse la alternativa entre caudillos locales y delegados reales, como se ha hecho a veces con respecto a determinados personajes, pues las circunstancias de la época hicieron compatibles (y necesarios) ambos papeles", BESG A MARRO Q UíN, A., "Domni Momi Comitis Bizcahiensis", Letras de Deusto 97 (2002), p. 20, nota 38.

13. PASTOR DÍAZ DE GARAYO, Castilla en el tránsito ..., p. 262.

14. Carazo también recibe mención tres veces, una renta nada desdeñable para una fuente poco prolija en cuanto a toponimia, en el Poema de Fernán González (traducción de ALARCOS LLO RACH, E., M adrid 1965, versos 171, 192, y 195). Además, en esta fuente se vuelve a relacionar a Carazo con Lara, y parece que este eje constituiría el núcleo originario de la familia condal castellano.

15. 14 referencias hasta 1054 de 52 posibles documentos, hemos descontado los cinco diplomas (\#246, 250, 261, 272 y 277) considerados falsos por M ARTín EZ DÍEZ, Colección documental del Monasterio de San Pedro de Cardeña. 
dad de Burgos y el río Arlanza. Aun así, se aprecia que su zona de influencia se centra en el cuadrante sur-oriental de la actual provincia de Burgos.

Unos años más tarde, ocurre una situación Ilamativamente análoga cuando el mismo espacio reaparece representado en negociaciones con el Reino de Pamplona por un magnate basado en Lara, y sin referencia directa al poder central castellano-leonés. Se trata también de una reafirmación, en este caso entre el conde Gonzalo Salvatórez (qui Laram dominabatur) y Sancho de Peñalén, y se confirma el acuerdo que garantizaba el libre acceso de peregrinos de la zona de Lara a San Millán de la Cogolla (Cogolla408, c. 1073). Estimamos que Lara aquí tiene una doble acepción: Ia plaza-fuerte en sí, dominada por Gonzalo Salvatórez; y una comarca, de donde provienen los referidos peregrinos. La trascendencia de este segundo acuerdo es quizás menor que la confirmación fronteriza que nos ocupa, pero demuestra la capacidad y disposición de los magnates de la comarca para tratar directamente con el vecino poder navarro.

Don Nuño también tiene una presencia diplomática en la documentación generada en las comarcas situadas al Norte del río Arlanzón y la Sierra de la Demanda, con varias apariciones en la corte de García de Nájera cuando ésta se reunía en Oña. ¿En qué capacidad estuvo Nuño Álvarez en O ña? Que actuara como embajador, apoyaría la hipótesis de que en Cogolla166 aparece como delegado del poder castellano, pero tenemos indicios de que no fuera así, y que su presencia en La Bureba se debía a intereses patrimoniales. Pues, si actuara como embajador, esperaríamos que apareciera también en la más numerosa diplomática del reinado de García conservada en la documentación riojana. Tenemos cuarenta diplomas de este período con escatocolos que sugieren la presencia de la corte, y los únicos cuatro que están conservados entre la documentación oniense son también los únicos donde aparece $\mathrm{D}$ on $\mathrm{Nuño}^{16}$. Más que una relación con la corte de García, sugerimos que la conexión de D on N uño sería con La Bureba, y que en las ocasiones en que visitaba sus heredades burebanas (en ese período bajo control navarro) se estimaría conveniente rendir homenaje al monarca navaro cuando éste visitaba O ña, lugar de enterramiento de su padre, Sancho III "el Mayor". En apoyo de esta hipótesis, notemos que en los contextos navarros el magnate castellano aparece en posiciones muy inferiores en la lista de confirmantes: no parece estar integrado en la jerarquía de la corte navarra, impresión confirmada por la extraña (y quizás algo soslayante) frase "isti sunt castellani".

Si entre 1037 y 1054 las apariciones de Nuño Álvarez en la documentación de Arlanza y de Cardeña son continuas, Ias referencias emilianenses a un señor llamado así caen casi todas fuera de estos límites cronológicos, con un especial protagonismo entre 1028 y 1031. Además, el N uño Álvarez que aparece en esta fuente siempre recibe el título "senior", un trato recibido una sola vez en otra

16. Cabe la posibilidad de que algunos escatocolos hayan sido abreviados por copistas posteriores, pero en los muy completos escatocolos reproducidos en la Colección Diplomática de La Rioja (\#4, del año 1044, por ejemplo) no hay mención alguna de N uño Álvarez. 
documentación (Arlanza49, 1048). Ya desde el momento de su estreno escatocolario en 1028 (Cogolla186) aparece codeándose con la élite de la corte de Sancho el Mayor, y el contexto geográfico de sus intervenciones sugiere un interés especial en la zona oriental de La Bureba. Teniendo en cuenta el desajuste geográfico, cronológico (aunque sea sólo cuestión de un lustro) y estilístico (Señor en vez de Don) entre las referencias emilianenses y las demás, ipodemos considerar que se trata del mismo individuo?

En cuanto a la diferencia estilística, Ernesto Pastor utiliza este mismo caso para ilustrar la equivalencia semántica entre los términos dominus y senior ${ }^{17}$. Tampoco debería preocuparnos demasiado el cambiante contexto geográfico ya que Arlanza54 demuestra la dispersión del patrimonio de Nuño Álvarez con posesiones en Ibeas de Juarros y quizás también en 0 ca $^{18}$ además de en Carazo y en Lara, y, como acabamos de sugerir, su presencia en la corte navarra en 0 ña se explica mejor a partir de intereses patrimoniales. Si, a partir de la muerte de Sancho el M ayor, lo vemos menos activo en la zona burebana, será porque se identifica claramente con Fernando de Castilla, y su patrimonio burebano se encuentra en el lado equivocado (desde el punto de vista de un miembro del séquito de Fernando I) de la frontera ${ }^{19}$. La adhesión a la corte castellana en sí sugiere que, aunque tuviera intereses patrimoniales muy dispersos, como parece ser el caso con muchos miembros de la clase magnaticia, el centro de su poder se ubicaría en torno a Carazo y no en La Bureba. Por lo tanto, y volviendo al percibido triple desajuste entre estas apariciones emilianenses de un Señor N uño Álvarez operativo en La Bureba y "nuestro" Don Nuño de Carazo, concluimos que los factores geográficos y cronológicos están íntimamente relacionados a los cambios geopolíticos del período, mientras el uso del apelativo navarro (senior) podemos asumir como una idiosincrasia diplomática emilianense.

\section{iv) En resumen}

Entre 1028 y 1054 las continuas referencias a Don Nuño Álvarez facilitan la construcción de una biografía relativamente coherente. Los escatocolos indican que fue uno de los magnates más importantes del Condado de Castilla durante todo este período, una impresión reforzada por su ostentación de la singular denominación "domno". Empieza a destacarse hacia finales del reinado de Sancho el Mayor,

17. PASTOR DÍAZ DE GARAYO, Castilla en el tránsito de la antigüedad al feudalismo, pp. 235-236.

18. Aunque la forma de la referencia "ribulo de Auca" quizás sugiere un microtopónimo local a la zona de Lara, pues Auka, Oca, etc. es un hidrónimo bastante extendido en esta parte de la península.

19. En otro trabajo hemos visto cómo incluso una institución de proyección transfronteriza -el monasterio de San Millán de la Cogolla- deja de participar tan activamente en el desarroIlo de sus posesiones burebanas cuando éstas se encuentran en el lado equivocado de la frontera entre 1054 y 1076, PETERSO N, D., La Sierra de la Demanda en la Edad M edia, Instituto de Estudios Riojanos, 2005, p. 82. 
siempre en el límite oriental de Castilla, zona donde también se concentraría su patrimonio. Pierde algo de protagonismo con la muerte de este monarca, pero tiene un creciente papel cortesano durante el segundo decenio del reinado de Fernando I. A la vez, sus posesiones burebanas le mantienen en contacto con la corte navarra, cuando ésta visita la Bureba, y en ocasiones el magnate castellano coincide con Fortún 0 choiz. Creemos que muere en la batalla de Atapuerca en 1054.

¿Cómo podemos reconciliar la abundante documentación del período 1028 a 1054 con la aislada referencia de 1016? La primera solución a considerar, y la más natural, sería la de dar por buena la participación de Nuño Álvarez en el tratado fronterizo de 1016, y aceptar que el mismo señor mantuviera su preeminencia entre 1016 y 1054. El problema entonces es la ausencia de otras referencias tempranas a tan importante magnate. Pues la distribución cronológica de referencias a Nuño Álvarez (una aparición de singular preeminencia, luego un silencio de doce años antes de una serie de constancia diplomática impresionante con una media superior a las dos referencias al $a n ̃ 0^{20}$ ) desafía cualquier lógica estadística. Más extraño todavía es que el momento de su máxima relevancia coincida con su estreno diplomático.

TABLA I. Referencias diplomáticas a D on Nuño Álvarez, 1000 a 1054

\begin{tabular}{|l|c|c|c|c|c|c|c|}
\hline Período & Cogolla & O ña & Cardeña & Arlanza & Sahagún & O tras $^{21}$ & TO TAL \\
\hline $1000-1004$ & 0 & 0 & 0 & 0 & 0 & 0 & 0 \\
\hline $1005-1009$ & 0 & 0 & 0 & 0 & 0 & 0 & 0 \\
\hline $1010-1014$ & 0 & 0 & 0 & 0 & 0 & 0 & 0 \\
\hline $1015-1019$ & 1 & 0 & 0 & 0 & 0 & 0 & 1 \\
\hline $1020-1024$ & 0 & 0 & 0 & 0 & 0 & 0 & 0 \\
\hline $1025-1029$ & 4 & 0 & 0 & 0 & 0 & 0 & 4 \\
\hline $1030-1034$ & 1 & $(2)^{22}$ & 1 & 0 & 0 & 0 & $2(+2)$ \\
\hline $1035-1039$ & 1 & 0 & 3 & 3 & 0 & 1 & 8 \\
\hline $1040-1044$ & 0 & 0 & 2 & 7 & 0 & 1 & 10 \\
\hline $1045-1049$ & 0 & 4 & 4 & 8 & 3 & 5 & 24 \\
\hline $1050-1054$ & 0 & 1 & $4(+1)$ & 2 & 0 & 1 & $8(+1)$ \\
\hline TOTAL & 7 & $5(+2)$ & $14(+1)$ & 20 & 3 & 8 & $57(+3)$ \\
\hline
\end{tabular}

20. 59 referencias en 26 años entre 1028 y 1054.

21. Recogidas de BLAN CO LOZANO, Colección diplomática de Fernando I. Esta colección incluye documentos de los archivos de las Catedrales de Astorga, León y Palencia, del monasterio aragonés de San Juan de la Peña, y el archivo diocesano de Santillana del Mar.

22. Entre paréntesis las referencias que aparecen en documentos considerados falsificaciones. 
U na alternativa sería que existiese un magnate Ilamado N uño Álvarez en el primer tercio del siglo XI, quizás pariente (¿abuelo?) del mismo que aparece tantas veces en la documentación posterior. Mientras tal cronología es posible, esta hipótesis tiene la misma debilidad que la expuesta anteriormente: la ausencia de otras referencias al mismo y aparentemente preeminente magnate en la documentación del primer cuarto del siglo. Ciertamente, la documentación cortesana durante este período es relativamente escasa, pero aun así, entre los 19 diplomas que existen se esperaría alguna referencia a un magnate de la preeminencia que Nuño Álvarez ostenta al firmar CogoIla166 ${ }^{23}$. Además esta hipótesis presupone que la denominación domno fuera hereditaria.

La tercera solución, y en nuestra estimación la más probable, es la de que el documento Cogolla166 es posterior a 1016, ya que éste es el único dato incongruente en una, por lo demás, coherente carrera de Don Nuño Álvarez entre 1028 y 1054.

\section{c) Fortún O choiz}

\section{v) La cronología de sus apariciones}

En contraste con los problemas causados por la homonimia a la hora de reconstruir la carrera de N uño Álvarez, el distintivo apellido de este señor hace relativamente fácil seguir su (irregular) trayectoria en la documentación navarra. A parece por primera vez en los escatocolos navarro-riojanos en el año 1013, como tenente de Viguera, y la última noticia que tenemos de él es de 1050. Por lo tanto, la incompatibilidad cronológica con el diploma de 1016 no es tan marcada como en el caso del magnate castellano, pues sí aparece en al gunos documentos anteriores a esa fecha crítica. Sin embargo, estas primeras apariciones son generalmente en posiciones escatocolarias secundarias. Dos diplomas onienses de escasa fiabilidad sugieren cierta prominencia hacia finales del reinado de Sancho el Mayor, pero no será en realidad hasta la segunda mitad del reinado de García de Nájera que aparece Fortún O choiz con cierta regularidad en posiciones escatocolarias prominentes.

23. Relación de escatocolos (1010-1028) en los cuales no aparece Nuño Álvarez: Condes64 (O ña8), 1011; Condes66 (O ña9), 1011; Condes67 (O ña10), 1011; Condes70 (O ña13), 1011; Condes71 (O ña14), 1011; Cogolla146, 1013; Condes74, 1014; Cogolla153, 1014; Cardeña220, 1019; Cogolla170, 1020; Valvanera1, 1020; Condes81, c. 1020; Cardeña224, 1024; Cardeña225, 1024; Condes79, 1024; Covarrubias13, 1024; Cogolla179, 1025; Covarrubias14, 1027; Cardeña228, 1028. 
TABLA II. Las primeras y generalmente discretas referencias a Fortún O choiz, indicamos la posición que ocupa en cada escatocolo

\begin{tabular}{|c|c|c|c|c|c|c|c|c|}
\hline 1013 & 1015 & 1016 & 1022 & 1024 & 1025 & $1030 ?^{24}$ & 1032 & 1032 \\
\hline \multirow[t]{2}{*}{ Cogolla146 } & Leire17 & Cogolla166 & Leire20 & Leire22 & Cogolla179 & O ña19 & Leire23 & Albelda33 \\
\hline & Leire18 & & & & & 0 ña26 & & \\
\hline 40 & $10^{0}$ & 1 & 50 & $4^{0}$ & $4^{0}$ & $2^{0}$ & 70 & 30 \\
\hline de 8 & de 13 & & de 7 & de 5 & de 7 & de 18 & de 8 & de 3 \\
\hline
\end{tabular}

En cambio, durante el primer decenio del reinado de García de Nájera (1035 a 1044) la participación de Fortún O choiz en la diplomática riojano-navarra se limita a dos ocasiones cuando se reunió casi toda la aristocracia del reino, y otras dos aisladas sesiones protocolarias en Leire y Albelda. En los dos escatocolos más completos del reinado aparece en lugares muy discretos, y en dieciocho diplomas cortesanos hasta finales de verano de 1045, sólo aparece cuatro veces.

TABLA III. Las escasas referencias a Fortún O choiz entre 1035 y 1044

\begin{tabular}{|c|c|c|c|}
\hline 1040 & 1042 & 1044 & 1044 \\
\hline Rioja3 & $\begin{array}{l}\text { Leire30 } \\
\text { Leire31 }\end{array}$ & Albelda35 & Rioja4 \\
\hline $\begin{array}{c}22 \underline{0} \\
\text { de } 35\end{array}$ & $\begin{array}{c}10 \\
\text { de } 8\end{array}$ & $\begin{array}{c}10 \\
\text { de } 3\end{array}$ & $\begin{array}{c}140 \\
\text { de } 24\end{array}$ \\
\hline
\end{tabular}

Sólo en el último lustro de su vida, su posición entre la élite de la corte navarra parece asegurada, y se consolida como tercer poder del reino sólo detrás del mismo rey, y de su ayo y confidente de toda la vida, Fortún Sánchez. En este período, además, contrae matrimonio con la hija de García, Doña Mencia ${ }^{25}$.

24. El testimonio de estos dos diplomas onienses merece muy poca credibilidad, y por lo demás el despegue diplomático se retrasa hasta 1042. Para ZABALZA DU QUE (Colección Diplomática de los Condes de Castilla, p. 556, nota 26) ambos diplomas onienses son falsos, y anteriormente habían levantado las sospechas de todo investigador que se acercaba a ellos: ÁLAMO, Colección diplomática de San Salvador de O ña, p. 41, nota 1; SÁNCHEZ CANDEIRA, Castilla y León en el siglo XI, p. 71, nota 20. A las reticencias de los autores citados añadimos la consideración de que estos diplomas no cuadran con la cronología de la carrera cortesana de Fortún 0 choiz.

25. Laturce2, 1049. Sabemos que el matrimonio se contrae en este último lustro por la escasa edad que tendría doña Mencia, que nació hacía 1030. 
TABLA IV. Consolidación de Fortún O choiz como tercer poder del reino 1045 a 1050

\begin{tabular}{|c|c|c|c|c|c|}
\hline 1045 & $1046^{26}$ & 1047 & 1048 & 1049 & 1050 \\
\hline Cogolla237 & $\begin{array}{l}0 \text { ña2/5 } \\
0 \text { ña32 } \\
0 \text { ña35 }\end{array}$ & Leire39 & $\begin{array}{l}\text { Albelda36 } \\
\text { Albelda37 }\end{array}$ & $\begin{array}{l}\text { Cogolla255 } \\
\text { Cogolla256 } \\
\text { Cogolla259 }\end{array}$ & $\begin{array}{l}\text { Cogolla267 } \\
\text { Cogolla269 }\end{array}$ \\
\hline $\begin{array}{c}20 \\
\text { de } 8\end{array}$ & $\begin{array}{c}20 \\
\text { de } 20 \text { ó } 25\end{array}$ & $\begin{array}{l}20 \\
\text { de } 17\end{array}$ & $\begin{array}{c}20 \\
\text { de } 9 \text { ó } 14\end{array}$ & $\begin{array}{c}20 \\
\text { de } 12,13 \text { ó } 14\end{array}$ & $\begin{array}{c}20 \\
\text { de } 11 \text { ó } 12\end{array}$ \\
\hline
\end{tabular}

Lo curioso de esta trayectoria es que, aun durante el período de menor actividad diplomática entre 1035 y 1044, Fortún 0 choiz mantiene el suficiente rango para resurgir del anonimato y ocupar puestos escatocolarios destacados, que por lo demás son el feudo exclusivo de los seguidores más fieles del rey: Fortún Sánchez, Sancho Fortúnez y Aznar Fortúnez. Además, como veremos a continuación, el magnate camerano se mantiene en la tenencia de Viguera durante todo el período estudiado, así que tampoco se trata de un exilio a otra tenencia marginada durante este período. La pregunta es esencialmente la misma que hemos planteado para N uño Álvarez, ¿cuál era la relación entre Fortún O choiz, su zona de influencia y la monarquía navarra?

\section{vi) El contexto geográfico}

Sólo conocemos un documento en el cual Fortún O choiz aparece como protagonista de una transacción inmobiliaria. Se trata de un diploma de García de Nájera de 1049 (Laturce2) en el cual se conceden a Fortún varias heredades en $\mathrm{N}$ alda, Leza y en Jubera, en los dos últimos lugares las heredades concedidas estando ya en posesión del magnate ("hodie sunt in tua potestate").

Por otra parte, durante la primera mitad del siglo XI es mucho más frecuente en la diplomática riojana-navarra que en la castellana que se indique la tenencia de los señores que aparecen como testigos y/o confirmantes. Así, con menos apariciones diplomáticas que Nuño Álvarez podemos estar seguros a la hora de vincular a Fortún 0 choiz con Viguera, fortaleza de gran relevancia estratégica en las sierras al sur de La Rioja, durante la totalidad de su protagonismo escatocolario. En los tempranos, y en al gunos respectos formalmente dudosos, diplomas procedentes de San Salvador de Leire ${ }^{27}$ aparece como dominator de Meltria, "castillo cerca de Viguera" todavía recordado en el microtopónimo Valdemetria ${ }^{28}$.

26. Estimamos que los tres diplomas 0 ña2/5, Oña32 y 0 ña35 (con fechas nocionales de 1043,1046 , y 1048 respectivamente), todos ellos referentes a la donación a San Salvador de 0 ña de un monasterio dedicado a San Juan cerca de Pancorbo y con grandísimas similitudes en cuanto a personal confirmante, tienen su origen en el mismo día y sesión protocolaria en 1046.

27. Leire17, 1015; Leire18, 1015; Leire22, c. 1024.

28. RO DRÍGU EZ R. DE LAM A, Colección Diplomática Medieval de la Rioja II, p. 360; PASCUAL MAYORAL, M.P., "Puente Caneco y castillo de Viguera (La Rioja) “, IV Semana de Estudios Medievales, Nájera 1993, p. 345. 
En 1040 tenemos una relación mucho más completa de su zona de influencia: "senior Fortuni Oxoiz cum ipsa terra que tenet, id est Bechera, ambabus Cambaribus, Ualdearneto cum omnibus villis Cantabriensis"29. La referencia a Cantabria se repite en la documentación de Leire y Albelda ${ }^{30}$. Lo más probable es que esta Cantabria corresponda al Cerro de Cantabria, otro punto de gran importancia estratégica que domina el vado que facilitaba el cruce del Ebro a la altura de la actual ciudad de Logroño, cerca de la desembocadura del Iregua, y donde se han encontrado restos de fortificaciones del siglo XI ${ }^{31}$. N otamos, además, que el Cerro de Cantabria se erige al norte del Ebro, y que, por lo tanto, en algunos períodos al menos, Fortún O choiz controlaría ambos lados de esta arteria. Las otras referencias parecen apuntar al control de una extensa parte del sector sur-oriental de la actual provincia de La Rioja. Bechera es una de las muchas ortografías que encontramos para Viguera, ambabus Cambaribus supondremos referirse a sendos Cameros (Nuevo y Viejo) y a los valles entre ellos, el del río Iregua, y el del río Leza. Si se añade el "Valle de Arnedo" (río Cidacos), casi todo el marco meridional de La Rioja estaría dominado por Fortún 0 choiz ${ }^{32}$.

En Cogolla166, sin embargo, se describe al magnate riojano como "de Pampilona". Sugerimos que esto se trataría sobre todo de una descripción genérica, lo mismo que a Don Nuño, quien sabemos de Carazo, se le describe con el genérico "de Castella". Tampoco deberíamos olvidar que éstas son las dos demarcaciones coronímicas utilizadas en el primer párrafo de Cogolla166 para identificar a las dos entidades delimitadas (creemos) en el tratado original y completo. Por lo tanto, las descripciones de Fortún O choiz y Nuño Álvarez como de Pampilona y Castella respectivamente serían un intento de relacionar este acuerdo parcial con el original, y así prestarle algo de autoridad. No creemos que Fortún fuera necesariamente de Pamplona, sino que los casi cuarenta años que le vemos relacionado con Viguera / M eltria (incluso cuando apenas figuraba en la diplomática riojano-navarra) sugieren que, como Nuño Álvarez, su tenencia coincidía con su base de poder patrimonial y probablemente ancestral.

29. Rioja3, 1040.

30. Leire23, 1032; Leire30, 1042; Leire31, 1042; Albelda35, 1044.

31. RODRÍGUEZ R. DE LAMA, Colección Diplomática Medieval de la Rioja I, p. 288; CEN ICEROS HERREROS, J., PÉREZ ARRO NDO, C. L., y TUD AN CA CASERO, J. M., "EI recinto medieval de monte Cantabria (Logroño, La Rioja)", Brocar 16, 1990, pags. 7-18.

32. PEÑA BO COS, "Alfoces y tenencias: La Rioja", p. 406, nota 80, también se repara en la extensión de esta tenencia, sugiriendo, además, que los varios seniores apellidados Fortunionis que aparecen en tenencias bajo-riojanas durante las décadas centrales del siglo XI podrían ser hijos de nuestro protagonista. Así Doña Mencia, la viuda de Fortún, aparece acompañada en 1057 por sus hijos Aznar, Iñigo, Sancho, Lope y Ximeno en un documento referente a Leza (Laturce4), y a continuación encontramos referencias a señores con estos nombres en los siguientes lugares: Monte Laturce (Laturce5, 1058); Arnedo y Cameros (Laturce7, 1063); M eltria (Laturce8, 1064), etc. 
vii) El Reino de Viguera, ¿enlace entre los Banu Q asi y Fortún O choiz?

Es llamativa la coincidencia antroponímica entre nuestro protagonista del siglo XI y muchos de los miembros de la familia Banu Q asi, como, por ejemplo, los dos hijos de Musa ibn Musa, "tercer rey de España", basados en La Rioja a finales del siglo IX: Lubb / Lope (= "Lobo" = "O choa") en Viguera / Arnedo; y Fortún en Tudela ${ }^{33}$. Q uizás esto no sea más que una mera coincidencia, y ya hemos comentado los problemas metodológicos causados por la homonimia antroponímica entre la aristocracia castellana. Sin embargo, existen otros indicios de continuidad en las sierras riojanas entre el período de dominio musulmán y la primera mitad del siglo XI. Sobre todo, observamos el mismo espacio fronterizo funcionando como una comarca diferenciada, y en cierta medida autónoma, a lo largo de más de dos siglos. En el contexto andalusí, Manzano Moreno insiste en la virtual independencia de las comarcas fronterizas del poder omeya, "una situación de facto en la que los diversos sectores fronterizos fueron divididos entre estas familias que pasaron a ostentar hereditariamente el señorío en estas zonas" ${ }^{\prime \prime 4}$, con los Banu Q asi como ejemplo estelar.

Aunque Cañada Juste aventura que el núcleo originario de los Banu $\mathbf{Q}$ asi podría estar al norte del río Ebro, entre O lite, Tudela y Ejea ${ }^{35}$, para cuando tenemos documentación detallada sobre esta familia, ya en la segunda mitad del siglo IX, sus operaciones se centran más al sur, en torno a las ciudades riojanas de Arnedo, Viguera y Calahorra ${ }^{36}$. Inevitablemente, si seguimos las fortunas de la familia a lo largo del siglo IX, habrá fluctuaciones en el espacio de influencia de los Banu Q asi, y en alza llegarían a controlar Huesca, Zaragoza e incluso Toledo, pero en esencia se concentraría en las sierras y valles de la Rioja Baja, y los nombres de Arnedo y Viguera se repiten con insistencia en los anales que relatan las gestas de la dinastía ${ }^{37}$.

La segunda manifestación histórica de este espacio es la enigmática aparición del Reino de Viguera en el último tercio del siglo X. Generalmente interpretado como una compensación a Ramiro Garcés, hermanastro del rey navarro Sancho Garcés "Abarca", por haberse quedado sin el trono principal, los datos

33. SÁN CHEZ ALBO RN OZ, C., O rígenes del Reino de Pamplona, Pamplona 1985, p. 177.

34. MANZANO MOREN O, La frontera de al-Andalus en época de los O meyas, pp. 361-364.

35. CAÑ ADA JUSTE, "Los Banu Q asi", p. 11. En realidad la base para está ubicación parece ser poco más que la búsqueda de un escenario entre las primeras apariciones cronísticas de esta saga -en Zaragoza en 788 y en Pamplona en 799.

36. MANZANO MORENO, La frontera de al-Andalus en época de los O meyas, pp. 117122, también añade Tudela, pero luego sugiere que esta última plaza sería, en origen, un enclave defensivo omeya contra los Banu Q asi.

37. Así CAÑADA JUSTE define a Arnedo como "el feudo más fiel y característico de los Banu Q asi", mientras el vínculo entre Lope ibn Musa y Viguera es particularmente pronunciado: reconstruye dicho castillo hacia 862; comete una matanza de los árabes de Zaragoza en un prado de Viguera en 874; y es enterrado en el mismo sitio en 875, CAÑADA JU STE, "Los Banu Q asi", pp. 41-49. 
sobre su extensión son muy escasos. La mayoría de las referencias son únicamente a Viguera, y no deberíamos suponer que equivaliera necesariamente al espacio antaño dominado por los Banu Q asi. Sin embargo, un diploma del Cartulario de San Martín de Albelda de 974 (Albelda26) describe a Ramiro como rey en Leza además de en Viguera. Parece lógico que un "reino", o por lo menos el territorio de un rex, porque así se le refiere siempre a Ramiro ${ }^{38}$, tuviera mayor envergadura que una tenencia cualquiera, y en este caso el hinterland camerano de Viguera parece el escenario más probable. Además, parece repetirse el régimen de semi-autonomía, ahora de Pamplona, antaño de los 0 meya, para este espacio plurisecularmente fronterizo.

Por último, volveremos a nuestro punto de arranque: el espacio controlado por Fortún 0 choiz, según la Carta de Arras de la Reina Estefanía (Rioja3, 1040), parece coincidir espacialmente tanto con el núcleo del dominio Banu Qasi, como con lo que intuimos ser el Reino de Viguera. Pero la similitud va más allá de lo espacial, pues también la relación de este magnate con la corte najerense, que hemos intentado reconstruir a través de los escatocolos, sugiere una comarca poderosa y autónoma, como habían sido antes el territorio de los Banu Q asi y el Reino de Viguera. En este respecto la distribución de las tenencias recordadas en Rioja3 es ilustrativa. La mayoría de los señores confirmantes aparecen con una o dos tenencias, y una de las pocas excepciones es Fortún Sánchez, mano derecha del rey García, que tiene a su nombre cinco plazas-fuertes, esparcidas a lo largo de la geografía riojano-navarra. Las posesiones de Fortún O choiz, en cambio, además de múltiples, son contiguas, y aparentemente extensivas, pues parecen describir comarcas ("ambabus Cambaribus et Valdearneto cum omnibus uillis Cantabriensis") más que plazas aisladas.

Tres manifestaciones históricas, por lo tanto, de lo que estimamos ser el mismo espacio: el núcleo patrimonial de los Banu Q asi en el siglo IX, el Reino de Viguera en el siglo X y el territorio controlado por Fortún 0 choiz en el siglo XI ${ }^{39}$. ¿Es una coincidencia que el mismo espacio se mantiene como una entidad fronteriza y semi-autónoma, o podemos enlazar dinásticamente a los últimos Banu Q asi, a los reyes de Viguera y a Fortún O choiz?

Según Al-U dri, se extinguió la dinastía de los Banu Q asi con la caída de la Rioja Alta en la década de los $920 \mathrm{~s}^{40}$. Sin embargo, conociendo los mecanismos

38. Por ejemplo, "Santione rex in Nagela et in Pampilona, et sub eius imperio parendo rex Ranimirus in U ekaria", Rioja2a, 972

39. Quizás deberíamos añadir una cuarta manifestación: el Señorío de los Cameros del siglo XII. En este caso, parece haber un enlace claro entre las posesiones de Fortún O choiz y las de los señores que consideramos sus descendientes, los varios caballeros apellidados Fortuniones que emergen en la Rioja Baja a partir de la segunda mitad del siglo XI, GARCÍA TURZA, Documentación medieval del Monasterio de San Prudencio de M onte Laturce (siglos X-XV), pp. 17-21; PEÑA BOCOS, "Alfoces y tenencias: La Rioja", p. 406, nota 80.

40. AL-'UD RI, Tarsi al-Ajbar, 75, p. 485, trad. Fernando DE LA GRANJA, "La M arca Superior en la obra de Al-'U drí" en Estudios de Edad Media de la Corona de Aragón, vol. VII, Zaragoza, 1967. 
de conquista y pactación del período, no parece descabellado pensar que un reducido territorio serrano quedara en manos de alguna estirpe menor de la ilustre familia muladí, y que en realidad este hipotético pacto contribuyese a la conquista cristiana de la Rioja Alta ${ }^{41}$. En este contexto resulta interesante la noticia que nos proporciona otro historiador árabe, Ibn Hazm, de la conversión al cristianismo de Fortún, un hijo menor del difunto Lope Ibn-M ohammed ${ }^{42}$. Sospechamos que, a cambio de esta apostasía y del reconocimiento de la soberanía del monarca navarro, este territorio camerano, último reducto de los Banu Q asi, conservaría cierta independencia.

No pretendemos que la hipótesis continuista esté libre de problemas e incógnitas. Si se aceptara la hipótesis de la pervivencia de un dominio residual de los Banu Q asi, ahora reconvertidos al cristianismo, dentro del Reino de Navarra ¿cómo y por qué se transformaría en el Reino de Viguera casi cincuenta años más tarde? ¿Cómo se explica la aparición en 941 (Albelda8) de un tenente en Viguera, Flaín Bermúdez, con onomástica que sugiere un origen castellano y nada que ver con los Fortún o Lope característicos de los Banu Q asi? ¿Si Fortún Sánchez era yerno de García Ramírez, como indica Cogolla270 (1050), por qué acabó el dominio camerano en manos de Fortún O choiz? ¿Será que éste fue marido de Fronilda, la otra hija (¿mayor?) de García Ramírez? ${ }^{43}$ En

41. Sobre la expansión territorial a base de pactos con poderes locales, con referencia a la expansión del Reino de Asturias por la cornisa cantábrica, vean BESG A M ARRO Q U ÍN, "Domni Momi Comitis Bizcahiensis", p. 20.

42. IBN HAZM, Yamharat ansab al-'arab, 8, 467, ed. Fernando DE LA GRANJA, "La Marca Superior en la obra de Al-'U drí", Zaragoza, 1967. En realidad, según Ibn Hazm, son dos primos carnales Banu Q asi, ambos Ilamados Fortún, que se convierten al cristianismo en la primera mitad del siglo $X$, uno hijo de Lubb ibn Muhammad, y el otro de su hermano y sucesor Abd Allah. Para un cuadro genealógico completo de los Banu Q asi, entre quienes la homonimia está tan extendida como entre los Álvarez de Castilla, vean MAN ZAN O M OREN O, La frontera de al-Andalus en época de los O meyas, p.427. Ibn Hazm relata aun más casos de apostasía entre Ios Banu Q asi, por ejemplo, Abd Allah e Ismail, ambos hijos de Mutarrif ibn Musa ibn M usa.

43. Según Laturce2, en 1049 Fortún O choiz estaría casado con doña Mencia, hija del rey García de Nájera, pero creemos que éste sería un segundo y tardío matrimonio, quizás después de un matrimonio entre Fortún y Fronilda. Sabemos que Fortún, tenente de Viguera ya en 1013, era mayor que el rey García, nacido hacia 1012. Por lo tanto, su matrimonio con la hija de éste, ya dos generaciones menor (nacida hacia 1030 en el mejor de los casos), sería tardío, y en toda probabilidad no las primeras nupcias del magnate. Q uizás detrás de este matrimonio habría un intento por parte de García de establecer una alianza con Fortún, fruto de la cual sería el retorno de Fortún a la corte najerense a partir de 1044, y a continuación la retoma cristiana de Calahorra en 1045. En este diploma García agradece a Fortún el buen servicio que le había prestado ("propter tuum bonum servitium quod michi fecisti").

Por otra parte, en 1057 (Laturce4) D oña M encia aparece haciendo una donación al monasterio de San Prudencio de M onte Laturce, y acompañada de sus hijos "don Aznar Fortúnez, testigo; y don Iñigo, testigo; y don Sancho, testigo; y don Lope, testigo; y don Ximeno, testigo" [GARCÍA TURZA, Documentación medieval del Monasterio de San Prudencio de Monte Laturce (siglos $X-X V)$, p. 29]. Sólo se explicita el patronímico de uno de estos hijos, pero la implicación es que sean todos Fortuniones ¿son todos los hijos de Dona M encia, o son algunos quizás 
fin, las incógnitas son tantas y la documentación tan parca que es imposible trazar una convincente línea dinástica entre los dos señores homónimos: el converso Fortún ibn Lubb y Fortún O choiz. Nos quedamos con la observación de la coincidencia onomástica y la semi-autonomía del mismo espacio fronterizo durante más de dos siglos.

\section{viii) En resumen}

En cuanto a la cronología, Fortún O choiz sí aparece diplomáticamente antes de 1016, pero siempre en papeles secundarios. No parece factible que este señor funcionara como único representante de la corte navarra en negociaciones con el Condado de Castilla en fecha tan temprana como 1016. Estimamos que su preeminencia cortesana no se hace realidad hasta el período anterior a la toma de Calahorra (c.1042-1044). Este acontecimiento bélico, y concretamente la ayuda que prestó Fortún a García de Nájera (las posesiones atribuidas a 0 choiz en Rioja3 prácticamente rodean Calahorra), luego apremiada con el matrimonio con Doña Mencia, son de suma importancia a la hora de entender su relación con el monarca navarro-najerense ${ }^{44}$. Sin embargo, su cronología es más confusa que la de Nuño Álvarez, ya que la diplomática apócrifa oniense hace posible un anterior protagonismo cortesano hacia finales del reinado de Sancho el Mayor, pero en ningún caso tan temprano como el pretendido 1016.

A lo largo del período estudiado, 0 choiz se vincula con la Tierra de Cameros, y por lo menos hacia finales del período controla un espacio de gran envergadura y no menos importancia estratégica, justo el rincón que linda con los dos vecinos más significativos de Navarra: Castilla y al-Andalus. Creemos que no se le debería considerar como un teniente más de la corona pamplonesa, pues su 'tenencia' es de un tamaño e importancia descomunal. La naturaleza singular de este dominio, resaltada por la aparición fugaz e insólita del Reino de Viguera, quizás tiene su origen en la relación entre los monarcas navarros y los 'últimos' y conversos Banu Q asi. Con esta apostasía se extinguió la dinastía a los ojos de los historiadores árabes, pero no necesariamente perderían su patrimonio en la Tierra de Cameros.

Volviendo a Fortún 0 choiz, la extensión, durabilidad e importancia estratégica de sus posesiones sugieren que el suyo sería un auténtico y, hasta cierto punto, autónomo poder regional, que parece mantenerse al margen de su relación con la corte. Así los dos magnates que reafirman la sección demandesa de

\footnotetext{
hijastros procedentes de otro hipotético matrimonio de Fortún O choiz? Q ue el matrimonio Fortún / M encia parece haber durado apenas un lustro (1044 a 1050), hace improbable que hubiese producido cinco hijos varones, pero además que los cinco hermanos Fortuniones aparecen ya en la documentación del entorno de Viguera-Albelda a partir de 1057 sugiere que no todos pueden ser hijos del matrimonio tardío entre Fortún y Mencia, sino de otro matrimonio anterior.

44. Aunque extrañamente no aparece en los primeros diplomas navarros que parecen celebrar esta victoria: Rioja6, 30 de Abril 1045; Cogolla235, 31 de Mayo 1045.
} 
la frontera navarro-castellana parecen tener poderío regional al margen de las concesiones y encargos del poder regio o condal.

\section{Conclusiones}

Si descartamos la fecha de 1016 es, sobre todo, porque las trayectorias de los dos firmantes no coinciden en ese momento con la preeminencia necesaria para actuar como representativos únicos de sus respectivos monarcas en la confirmación de tan importante documento. Si añadimos otros elementos internos del texto (la ausencia de formalismos propios de un producto canciIleresco, los tiempos verbales empleados, y el hecho de que sólo se delimita una parte de la frontera navarro-castellana) llegamos a la conclusión de que el texto Cogolla166 es una ratificación parcial, posterior y en probabilidad de iniciativa particular.

Pero, ¿qué cronología alternativa proponemos para tal ratificación? Esto ya es más complicado, pues las incógnitas son muchas. En primer lugar, la diplomática apócrifa oscurece la cronología de los dos magnates protagonistas, aunque de todos modos 1016 es una imposibilidad. Algún momento entre 1028 y 1054 sería la probable fecha de la reafirmación parcial, pero es difícil precisar más. Si buscamos un momento de inestabilidad fronteriza entre Castilla y Navarra en que encajar el acuerdo tampoco avanzamos mucho, ya que esta circunstancia es prácticamente una constante durante el período que nos ocupa. Empezando con la muerte del conde Sancho en 1017, pasamos a un período de debilidad institucional en Castilla durante el condado del infante García Sánchez, 1017-1029. El último lustro de este período vería una creciente participación de Sancho el Mayor en Castilla, hasta el punto de asumir control del Condado a partir de 1029, y posiblemente algo antes. Tampoco resolvería las tensiones la muerte del gran monarca navarro y la tan comentada división de su imperio entre sus hijos, como demuestra la batalla fratricida de Atapuerca (1054).

O tra pista podría ser la falta de una referencia en Cogolla166 a otros monarcas que los dos Sanchos. Aunque discernimos una capacidad y disponibilidad por parte de los dos magnates confirmantes a actuar por su cuenta, la tendencia en la diplomática altomedieval es a reconocer, por lo menos nominalmente, a la autoridad superior, aunque el caso prestado a ella podría ser mínimo. Esta consideración hace difícil una cronología posterior a 1035, ya que supondría un desplante extraordinario hacia García de Nájera y Fernando de Castilla, teniendo en cuenta la trascendencia potencial del texto. Por lo tanto, nos inclinamos hacia una reafirmación durante los últimos años del reinado de Sancho el Mayor, aunque sin desechar del todo la hipótesis de un acuerdo magnaticio en rebeldía. Sabemos que García de Nájera experimentó serios problemas con parte de su aristocracia en los años anteriores a la batalla de Atapuerca, y notamos que Fortún O choiz desaparece de la diplomática a partir de 1050, cir- 
cunstancia que hemos atribuido a su muerte, pero que también podría explicarse por desavenencias con el monarca navarro ${ }^{45}$.

Sin embargo, nuestra intención principal aquí ha sido la de cuestionar la fecha hasta ahora atribuida al texto, incompatible con las biografías de los dos magnates confirmantes. Lamentamos no poder ofrecer una fecha alternativa precisa, ya que no sabemos en qué circunstancias y en qué espíritu se decidió reafirmar el status quo territorial, un hecho insólito, por lo menos en cuanto al testimonio diplomático observado.

¿Cuál es la relación entre estos señores y los espacios que controlan? Sugerimos que va más allá de lo que correspondería a un mero delegado del poder central. Pues la relación con el mismo espacio es duradera, aparentemente hereditaria, y en cierta medida independiente de las cambiantes circunstancias cortesanas. Esta evaluación se hace sobre todo en referencia a Fortún 0 choiz y la semi-autonomía durante varios siglos de una comarca centrada en la Sierra de Cameros. En el caso de Nuño Álvarez, su independencia de Fernando I es menos evidente. Sin embargo, su anterior acercamiento a la corte navarra (c.1028) parece demostrar la independencia operativa de un magnate fronterizo cuando el poder central se debilitaba, caso del condado castellano entre 1017 y 1028.

45. También el comportamiento diplomático de Nuño Álvarez se altera en este periodo. A partir de 1047 sus apariciones en diplomas de factura cortesana empiezan a escasear, sobre todo cuando se introduce el matiz de la homonimia y se eliminan los varios diplomas que los expertos en esta materia [BLANCO LOZANO, Colección diplomática de Fernando I (10371065); y MARTín EZ DíEZ, Colección documental del Monasterio de San Pedro de Cardeña] consideran de autenticidad sospechosa, sólo queda un diploma regio con indiscutible presencia de Don Nuño Álvarez, y curiosamente aparece en la corte de García en O ña. Este periodo coincidiría con la ausencia diplomática de Fortún O choiz a partir de 1050, y por lo tanto es posible que en el lustro anterior a la batalla de Atapuerca ambos magnates confirmantes se retirasen de sus respectivos ambientes cortesanos, y de allí podría venir el desplante de no citar a los belicosos hermanos monarcas, en su reafirmación particular de su sector de la frontera. 